\title{
Percutaneous Radiofrequency Ablation of Small (1-2 cm) Hepatocellular Carcinomas Inconspicuous on B-Mode Ultrasonographic Imaging: Usefulness of Combined Fusion Imaging with MRI and Contrast-Enhanced Ultrasonography
}

\author{
Min Woo Lee, ${ }^{1,2}$ Hyo Keun Lim $\mathbb{D}^{1,2}$ Hyunchul Rhim, ${ }^{1,2}$ Dong Ik Cha, ${ }^{1}$ Tae Wook Kang, \\ Kyoung Doo Song, ${ }^{1}$ Ji Hye Min, ${ }^{1}$ Geum-Youn Gwak, ${ }^{3}$ Seonwoo Kim, ${ }^{4}$ and David S. K. Lu ${ }^{5}$ \\ ${ }^{1}$ Department of Radiology and Center for Imaging Science, Samsung Medical Center, \\ Sungkyunkwan University School of Medicine, Seoul 06351, Republic of Korea \\ ${ }^{2}$ Department of Health Sciences and Technology, SAIHST, Sungkyunkwan University, Seoul 06351, Republic of Korea \\ ${ }^{3}$ Department of Medicine, Samsung Medical Center, Sungkyunkwan University School of Medicine, Seoul 06351, Republic of Korea \\ ${ }^{4}$ Statistics and Data Center, Samsung Medical Center, Seoul 06351, Republic of Korea \\ ${ }^{5}$ Department of Radiological Sciences, David Geffen School of Medicine at UCLA, 757 Westwood Plaza, Los Angeles, CA 90095, USA
}

Correspondence should be addressed to Hyo Keun Lim; rfalim@skku.edu

Received 3 May 2018; Accepted 4 June 2018; Published 14 June 2018

Academic Editor: Michele Molinari

Copyright (C) 2018 Min Woo Lee et al. This is an open access article distributed under the Creative Commons Attribution License, which permits unrestricted use, distribution, and reproduction in any medium, provided the original work is properly cited.

\begin{abstract}
Purpose. To assess usefulness of adding contrast-enhanced ultrasonography (CEUS) to fusion imaging (FI) for percutaneous radiofrequency ablation (RFA) of hepatocellular carcinomas (HCCs) inconspicuous on FI alone. Therapeutic outcomes of RFA under CEUS-added FI guidance for HCCs inconspicuous on FI alone were also evaluated. Methods. This prospective study was approved by the institutional review board and informed consent was obtained from all patients. Planning US was performed with FI for 126 patients with a single HCC $(1-2 \mathrm{~cm})$ to evaluate the feasibility of RFA by grading lesion conspicuity score using a four-point scale. RFA was performed under CEUS-added FI guidance for HCCs inconspicuous on FI alone. We evaluated how many HCCs initially inconspicuous on FI became conspicuous after adding CEUS. After CEUS-added FI-guided RFA, therapeutic outcomes including rates of technical success, primary technique efficacy, major complications, and local tumor progression were assessed. Results. After adding CEUS, 90.5\% (19/21) of all tumors initially inconspicuous on FI became conspicuous, thus enabling direct targeting for RFA. Technical success and primary technique efficacy rates were $94.7 \%$ (18/19) and 100\% (19/19), respectively. No major complications were observed after RFA. Cumulative local tumor progression rates after RFA were estimated to be 5.3\%, $10.8 \%$, and $10.8 \%$ at 1,2 , and 3 years, respectively. Conclusion. Adding CEUS to FI is useful for improving the conspicuity of HCCs inconspicuous on FI alone, thus enabling successful percutaneous RFA with excellent therapeutic outcomes.
\end{abstract}

\section{Introduction}

It can be challenging to ablate small hepatocellular carcinomas (HCCs) under B-mode ultrasonography (US) guidance as small HCCs sometimes have poor sonographic conspicuity [1]. Fusion imaging (FI) of B-mode US and pre-acquired computed tomography $(\mathrm{CT}) /$ magnetic resonance imaging (MRI) has emerged as a useful guidance modality for percutaneous radiofrequency ablation (RFA) of small HCCs [2-5]. FI increases the confidence of a subtle lesion by colocalizing it to pre-acquired CT/MRI. However, when a tumor is completely isoechoic compared to surrounding normal liver, FI can only estimate the location of what is in essence a "virtual target," and incomplete ablation can occur even after FI-guided RFA [6]. This shortcoming is due to the inherent registration error that occurs when applying rigid registration to a deformable organ during motion.

Contrast-enhanced US (CEUS) using Sonazoid (gaseous perflubutane, GE Healthcare, New York City, NY) has emerged 
as a promising technique for localizing small HCCs and guiding percutaneous RFA $[7,8]$. HCCs can be localized with high confidence using the post-vascular phase unique to Sonazoid [9].

CEUS is now often combined with FI for percutaneous RFA of challenging small HCCs $[10,11]$. This combination has been shown to enhance the feasibility of percutaneous RFA for HCCs inconspicuous on FI alone [11, 12] and also better assess the ablation zone [13]. However, most of these studies had retrospective designs. They included heterogeneous mixtures of liver tumors and only evaluated the value of CEUSadded FI for localizing liver tumors without sufficient followup data $[11,12,14,15]$. To our knowledge, there are no solid data regarding how many tumors initially inconspicuous on FI become conspicuous after adding CEUS, thus enabling direct targeting for RFA.

Therefore, the primary endpoint of this trial was to assess the value of adding CEUS to FI for improving lesion conspicuity and the technical feasibility of percutaneous RFA of HCCs $(1-2 \mathrm{~cm})$ inconspicuous on FI alone. The secondary endpoint was to assess the therapeutic outcomes of RFA under CEUS-added FI guidance for HCCs inconspicuous on FI alone.

\section{Materials and Methods}

2.1. Sample Size Estimation. The detection rate of very-earlystage HCC [Barcelona Clinic Liver Cancer (BCLC) stage 0 , single nodule $\leq 2 \mathrm{~cm}$ ] on FI has been reported to be approximately $85 \%$ [16]. Assuming that $15 \%$ are discordant pairs analyzed using the exact binomial test, a sample size of 101 is required with a 5\% significance level and $80 \%$ power. Considering a drop-out rate of $20 \%$, the minimum sample size was determined to be 126 .

2.2. Patients. This prospective study was approved by the Institutional Review Board of Samsung Medical Center, a tertiary referral center. Informed consent was obtained from all patients prior to enrollment. Between August 2013 and January 2015, a total of 126 patients satisfying the following inclusion criteria underwent planning US to assess the feasibility of percutaneous RFA in our department. Inclusion criteria were as follows: (a) a treatment-naïve, single HCC 1-2 $\mathrm{cm}$ in diameter on contrast-enhanced MRI with gadoxetic acid (Gd-EOB-DTPA), (b) classification as Child-Pugh class A or B, (c) normal coagulation status, and (d) absence of vascular invasion and extrahepatic metastasis on MRI at the time of diagnosis. Exclusion criteria were as follows: (a) patients who did not undergo CEUS despite inconspicuous tumors on FI, (b) artificial ascites used instead of CEUS to improve sonographic window, (c) RFA infeasible due to reasons other than lesion conspicuity, (d) other treatments performed instead of RFA, and (e) patients who did not agree to participate in this study or who were lost to follow-up.

Treatment modality was determined based on recommendations from a multidisciplinary tumor board. Among these 126 patients, 34 patients were determined to have HCCs inconspicuous on FI alone; 19 of these patients who finally underwent percutaneous RFA under CEUS-added FI

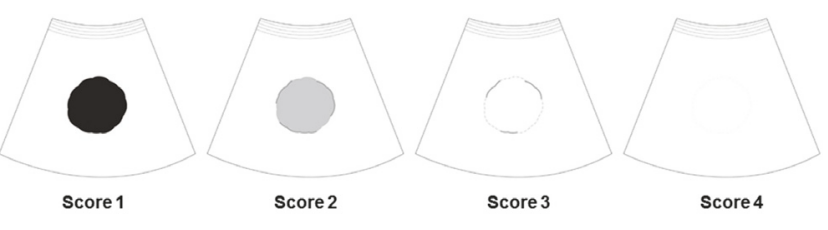

FIGURE 1: A schema showing the conspicuity score.

guidance were included in the evaluation of therapeutic outcomes after RFA.

Diagnosis of HCC was based on typical imaging features according to the American Association for the Study of Liver Disease guidelines [20] obtained on MRI. Scans were acquired using a 3.0-T system (Intera Achieva 3.0-T, Philips Healthcare, Best, The Netherlands) equipped with a 32-channel phased-array coil.

2.3. Planning US with Fusion Imaging. Planning US was performed to evaluate whether percutaneous RFA was feasible by one of three experienced radiologists (H.K.L., H.R., and M.W.L. with 15, 15, and 10 years of clinical experience with percutaneous RFA, respectively). For planning US, FI (Volume Navigation; GE Healthcare, Waukesha, WI) of the LOGIQ E9 system (GE Healthcare) was routinely used with a $1-5-\mathrm{MHz}$ convex probe regardless of the initial US conspicuity of the tumor. The process of image fusion between B-mode US and MRI was similar to the methods of previous studies $[2,16]$. After image fusion, the HCC conspicuity score on Bmode US was graded by the radiologist performing planning US using a four-point scale at the time of US examination (Figure 1 and Table 1). Lesions with a score of 1 or 2 were regarded as conspicuous HCCs, while those with a score of 3 or 4 were regarded as inconspicuous HCCs. The technical feasibility of RFA was also evaluated based on the expected electrode path, adjacent organ vulnerable to thermal injury, and heat-sink effect [17].

2.4. Percutaneous RFA. Percutaneous RFA was performed using a 200-W RF generator (VIVA RF system; STARmed, Goyang, Korea) and a length-adjustable RF electrode (Proteus; STARmed) by the radiologists who performed planning US. Additional CEUS was applied for HCCs inconspicuous on FI to increase lesion conspicuity, and RFA was performed if feasible under CEUS-added FI guidance. When CEUS was added to FI, image fusion between B-mode US and MRI was first performed to estimate the location of the index tumor before applying CEUS. Then, the US image was switched from B-mode to CEUS mode and displayed side by side with the fused MRI. Contrast harmonic imaging technique with a default setting of a mechanical index level of 0.24 was used. The focal zone was placed in the posterior margin of the liver. Sonazoid was injected intravenously at a dose of $0.015 \mathrm{~mL} / \mathrm{kg}$, followed by a flush with $10 \mathrm{~mL}$ of normal saline. Images were obtained during each phase: arterial (10-40 seconds after contrast injection), portal (60-90 seconds), and delayed phase ( 3 minutes) and post-vascular phase (more than 10 minutes) [11]. If the index tumor was not clearly identified on the arterial phase but was visible on the post-vascular 
TABLE 1: Scoring criteria for evaluation of tumor conspicuity.

Score
$\begin{array}{r}\text { The echogenicity of the index tumor is definitely different from that of the surrounding liver and more than } \\ 90 \% \text { of the tumor has a well-defined margin }\end{array}$
$\begin{array}{r}\text { The echogenicity of the index tumor is slightly different from that of the surrounding liver and more than } 50 \% \\ \text { of the tumor has a well-defined margin }\end{array}$
The index tumor is nearly isoechoic to the surrounding liver and less than $50 \%$ of the tumor has a well-defined
margin

This scoring system was described in our previous study [11] and was established after reviewing previous relevant studies regarding planning US for RFA and making necessary updates [17-19].

phase, Sonazoid was reinjected in the post-vascular phase to visualize arterial enhancement of the target lesion [9]. The conspicuity score of the index tumor after adding CEUS was reassessed by the aforementioned scoring system based on the post-vascular phase as the temporal window of the postvascular phase is long and the RF electrode is inserted during the phase. When the index tumor was definitely unidentifiable even after adding CEUS, percutaneous RFA was not attempted. Instead, an alternative treatment or imaging follow-up was considered.

The technical goals of RFA were to eradicate the tumor and to achieve a minimum $0.5-\mathrm{cm}$ ablative margin where feasible. The energy deposition algorithm was based on the manufacturer's recommended protocol. Overlapping ablation was performed when needed. Although the standard ablation time recommended by the manufacturer was 12 minutes, RFA was finished earlier when the echogenic zone created was large enough to achieve a sufficient ablative margin or collateral thermal injury was suspected. The electrode path was cauterized during electrode removal at the end of the ablation session to avoid tract bleeding or tumor seeding.

\subsection{Assessment of the Effects of Adding CEUS to FI and the} Therapeutic Efficacy of RFA. For tumors inconspicuous on FI alone, we evaluated how initially inconspicuous HCCs became conspicuous after adding CEUS to FI, thus enabling direct targeting of percutaneous RFA. The rate of conspicuous HCCs on FI was compared with that on CEUS-added FI. Lesions conspicuous on FI alone were also regarded as conspicuous on CEUS-added FI, even though additional CEUS was not performed.

For patients who underwent RFA, therapeutic efficacy and complications were assessed using multiphase liver CT obtained within 12 hours after RFA. In addition, chest radiography, multiphase liver CT or MRI, and laboratory tests including serum $\alpha$-fetoprotein were performed one month after initial discharge and every 3-4 months thereafter. Assessments were performed according to standardized terminology and reporting criteria for image-guided tumor ablation [21]. Technical success was defined as when the index tumor was treated according to our treatment protocol and was covered by ablation zone completely on the immediate post-RFA CT images [21]. The treatment course included all RFA sessions performed within one month to eradicate any residual tumor. Primary technique efficacy was assessed based on follow-up CT images taken one month after ablation. Local tumor progression (LTP) was defined as when foci of untreated disease appeared in tumors that were initially considered to be completely ablated on follow-up CT or MRI. A major complication was defined as any event that resulted in substantial morbidity and disability, an increased level of care, or substantial lengthening of hospital stay [21]. All other complications were regarded as minor.

2.6. Statistical Analysis. The conspicuity scores of inconspicuous HCCs on FI alone before versus after adding CEUS were compared using the Wilcoxon signed rank test. The rate of conspicuous HCCs on FI alone was compared with the rate of conspicuous HCCs on CEUS-added FI. Improvement of conspicuity score with the addition of CEUS was tested with the exact binomial test. Cumulative LTP rates of CEUSadded FI-guided RFA were estimated using the Kaplan-Meier method. All statistical analyses were performed using the SPSS Statistics software package (version 18.0; SPSS, Chicago, IL). A $p$ value less than 0.05 was considered statistically significant.

\section{Results}

3.1. Patient and Lesion Characteristics. Of 126 patients, 92 (73.0\%) patients had a lesion conspicuity score of 1 or 2 , whereas $34(27.0 \%)$ patients had a conspicuity score of 3 or 4 on FI (Figure 2). Of the 34 patients with a score of 3 or 4, 13 did not undergo CEUS due to the following reasons: percutaneous RFA was infeasible due to reasons other than lesion conspicuity $(n=7)$; artificial ascites fluid was used to enhance lesion conspicuity $(n=4)$; or other treatments were performed instead of percutaneous RFA $(n=2)$. The remaining 21 patients with conspicuity score of 3 or 4 also underwent CEUS (Figure 2). The conspicuity score of the 21 HCCs (mean \pm standard deviation [SD], $1.2 \pm 0.2 \mathrm{~cm}$; median, $1.1 \mathrm{~cm}$; range, $1.0-1.8 \mathrm{~cm}$ ) was significantly higher after adding CEUS (before CEUS; median, 3; range, 3-4 versus after CEUS; median, 1 ; range, $1-4 ; p<0.001$ ) (Figure 3). Of the 21 lesions inconspicuous on FI alone, 19 (90.5\%) became conspicuous after adding CEUS (16 on both arterial and post-vascular phase, two in post-vascular phase, and one in arterial phase) (Table 2). The rate of conspicuous HCCs on CEUS-added FI was significantly higher than that on FI alone [(92+19)/(92+21), $98.2 \%$ versus $92 /(92+21), 81.4 \% ; p<0.001]$. 


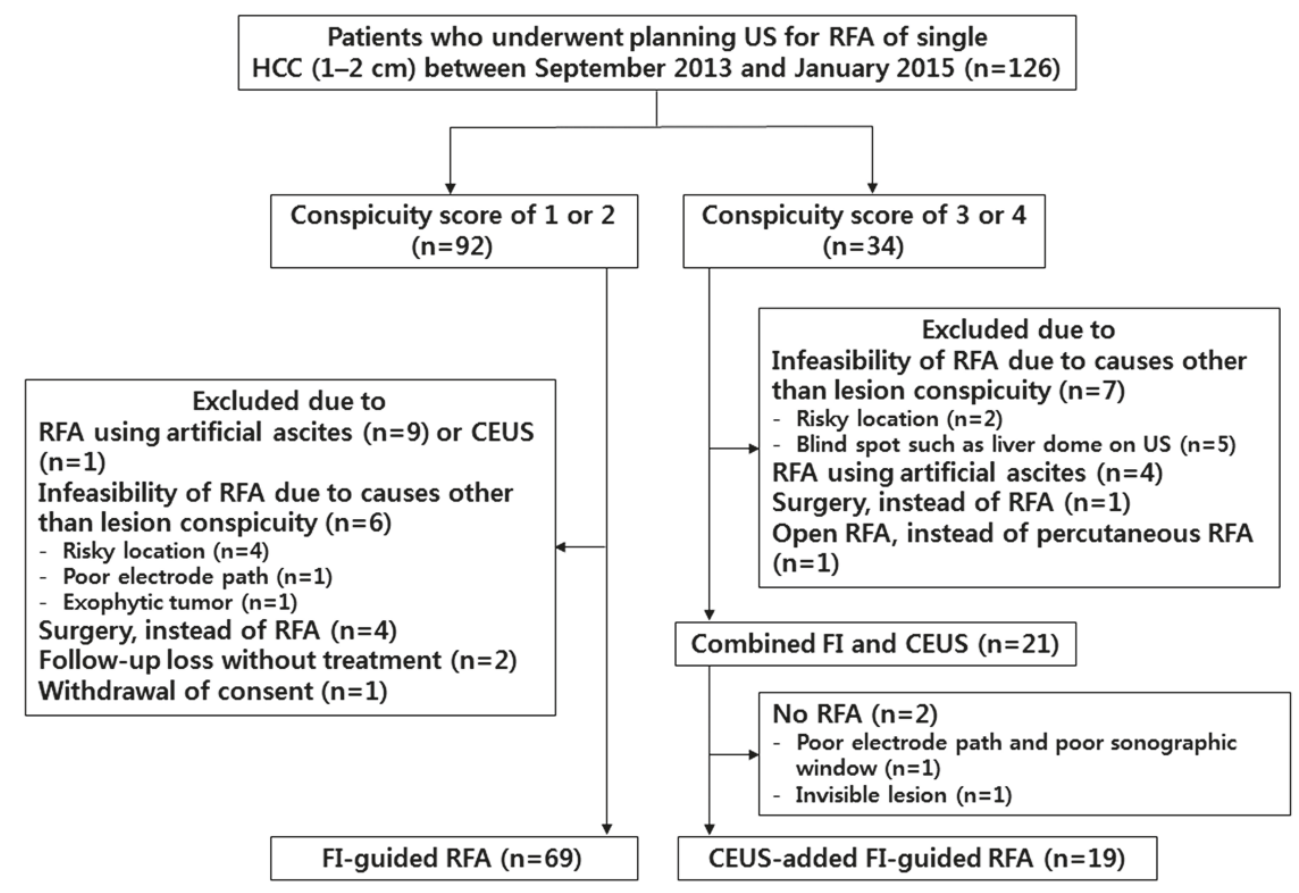

FIGURE 2: Flow diagram of the patient enrollment process. US = ultrasonography; HCC = hepatocellular carcinoma; RFA = radiofrequency ablation; FI = fusion imaging; CEUS = contrast-enhanced ultrasonography.

TABLE 2: Conspicuity score before and after CEUS in the 21 patients with fusion imaging-inconspicuous HCCs.

\begin{tabular}{lc}
\hline Before CEUS & After CEUS \\
\hline $3(n=13)$ & $1(n=8)$ \\
& $2(n=4)$ \\
& $3(n=1)$ \\
$4(n=8)$ & $1(n=4)^{\mathrm{a}}$ \\
& $2(n=3)^{\mathrm{b}}$ \\
& $4(n=1)^{\mathrm{c}}$ \\
\hline
\end{tabular}

CEUS $=$ contrast-enhanced ultrasonography; HCC = hepatocellular carcinoma.

${ }^{a}$ Residual tumor was noted after the first RFA session in one patient in whom the tumor was located in the subcapsular area in segment 5 , just below the right rib. Technique efficacy was achieved after the second RFA session.

${ }^{\mathrm{b}}$ In one patient, although the lesion was identified after CEUS was added, both the expected electrode path and sonographic window were poor. Therefore, chemoembolization was performed instead of percutaneous RFA. ${ }^{c}$ In this patient, the target lesion was not identified on the arterial or postvascular phase. Therefore, instead of treatment, this patient was followed up with CT/MRI. Eventually, the lesion disappeared on 8-month follow-up MRI, indicating that it was a pseudolesion.

Among the 21 patients who underwent CEUS-added FI, one underwent chemoembolization instead of RFA due to poor electrode path and poor sonographic window due to perihepatic fat thickness, even though the index tumor was identified after adding CEUS (conspicuity score: 2). Another patient did not undergo RFA due to invisibility of the target lesion, even after adding CEUS. The latter patient was followed up with CT/MRI and the lesion had disappeared on 8-month follow-up MRI, indicating that it had been a pseudolesion. Eventually, direct targeting for percutaneous RFA was possible in $90.5 \%$ (19/21) of tumors initially inconspicuous on FI alone after adding CEUS (Figure 2).

3.2. Percutaneous RFA and Its Therapeutic Outcomes. The baseline characteristics of the 19 patients who underwent RFA under CEUS-added FI guidance are summarized in Table 3. Tumor size was $1.2 \pm 0.2 \mathrm{~cm}$. After RFA, a residual tumor was detected in one patient at CT, requiring a second ablation session to complete the treatment course. In this patient, the tumor (1.2 cm in diameter) was located in the subcapsular area of segment 5, just below the right rib. Although the conspicuity score of the tumor increased from 4 to 1 after adding CEUS, the sonographic window was poor due to the overlying rib shadow.

Meanwhile, incomplete ablation was avoided by adding CEUS in one case where a subtle lesion on FI was initially thought to be a true lesion that corresponded to the tumor nodule on MRI (Figure 4).

The therapeutic outcomes are summarized in Table 4. The technical success rate was $94.7 \%$ (18/19). No major complications were identified after CEUS-added FI-guided RFA. No residual tumors were identified in any patient on CT obtained one month after RFA (primary technique efficacy rate: $100 \%, 19 / 19)$. The cumulative LTP rates after CEUSadded FI-guided RFA were estimated to be $5.3 \%, 10.8 \%$, and $10.8 \%$ at 1,2 , and 3 years, respectively.

\section{Discussion}

In general, FI can enhance the detectability of small HCCs inconspicuous on B-mode US and reduce false-positive 


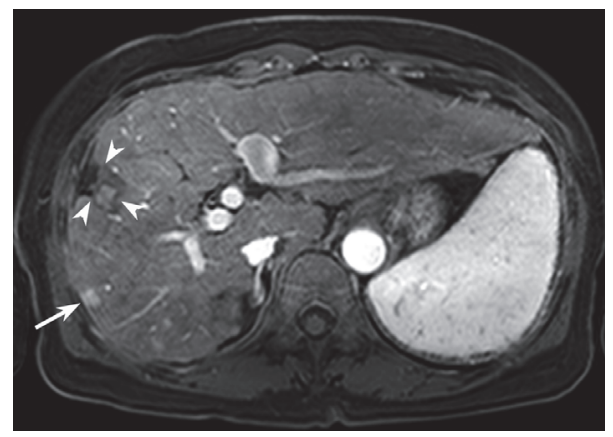

(a) Arterial-phase MRI showing a small hypervascular HCC (arrow) in segment 5 of the liver. Arrowheads indicate a previous RFA zone

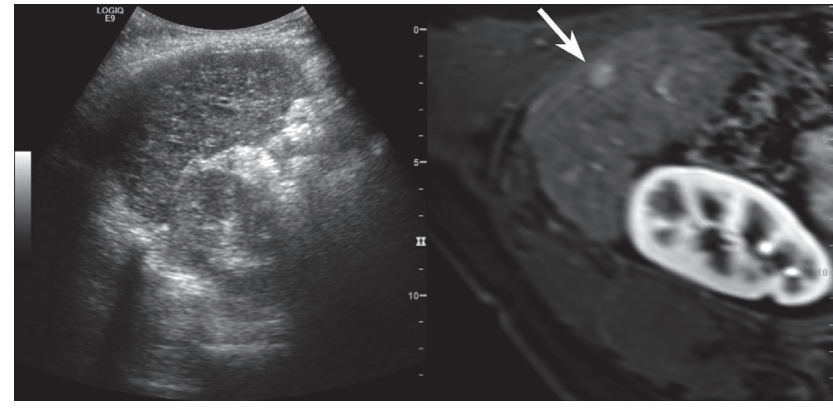

(b) After fusion imaging, the HCC nodule could not be identified on B-mode US imaging at the corresponding site on fused MRI (arrow). Therefore, the index tumor was given a conspicuity score of 4 on fusion imaging as it was definitely unidentifiable

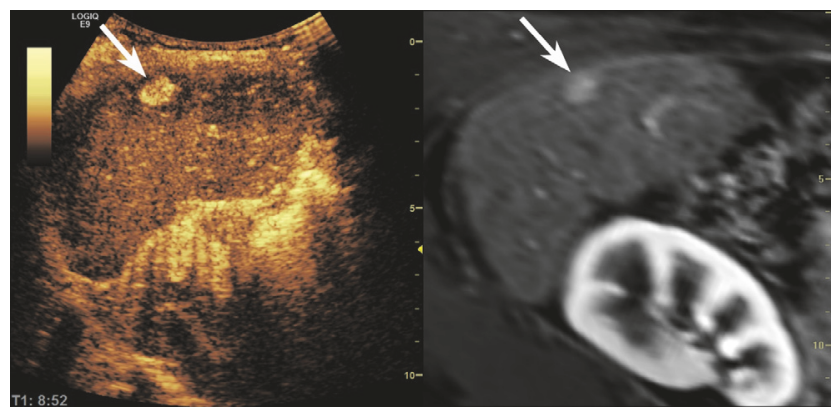

(c) On arterial-phase imaging obtained after the second injection of Sonazoid, a small enhanced lesion (arrow) was clearly identifiable at the corresponding location on fused MRI. After the first injection of the contrast agent, the optimal arterial phase was not obtained due to irregular respiration of the patient

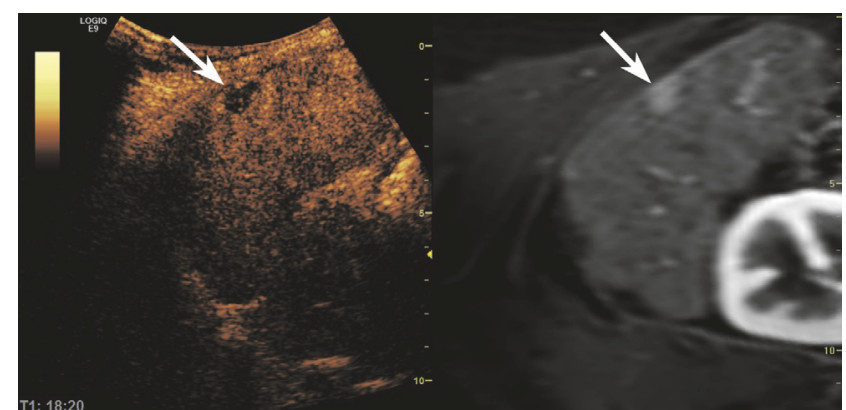

(d) On post-vascular-phase imaging, the index tumor (arrow) was clearly identified as a perfusion defect at the corresponding site (arrow) on fused MRI. Therefore, the index tumor was given a conspicuity score of 1 on CEUSadded fusion imaging

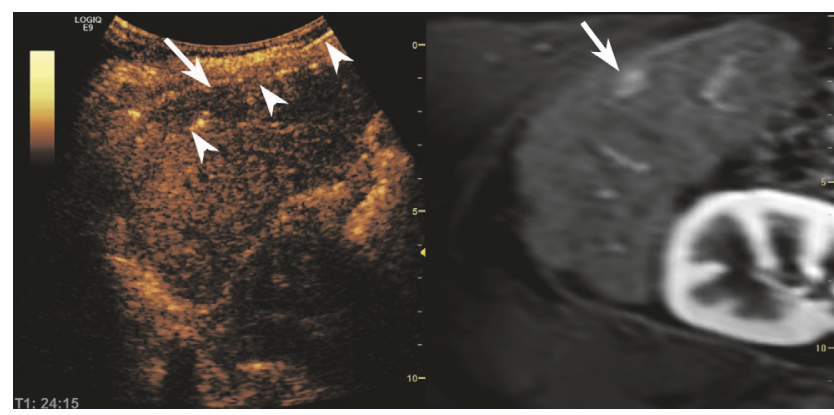

(e) An RF electrode (arrowheads) was inserted into the index tumor (arrow) under CEUS-added fusion imaging guidance, after which RF energy was applied

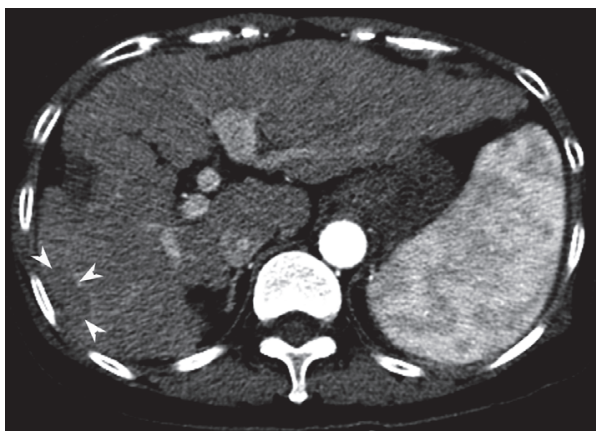

(f) Arterial-phase CT image obtained immediately after RFA revealed technical success with sufficient ablative margins (arrowheads). LTP was not identified on follow-up MRI obtained 32 months after RFA (not shown here)

FIGURE 3: Images from a 59-year-old woman with a 1.1-cm HCC and hepatitis B virus-related liver cirrhosis who had previously undergone percutaneous RFA of HCC.

detection of HCCs on B-mode US [16, 22]. In a previous study [16], false-positive detection on B-mode US was as high as 9.1\% (9 of 99) for HCCs (1-2 cm) based on FI findings. In this context, we have adopted FI as the routine guiding modality for percutaneous RFA of HCCs. However, FI is not always sufficient for localizing small HCCs. Actually, 31.3\% (76/243) of HCCs (mean size, $1.5 \mathrm{~cm}$; range, $0.3-5.0 \mathrm{~cm}$ ) were invisible on FI in a recent study [5].
Our study demonstrated that adding CEUS to FI improved the conspicuity of HCCs inconspicuous on FI of real-time US and MRI (Table 2). Consequently, it enabled percutaneous RFA of HCCs inconspicuous on FI alone in most cases $(90.5 \%, 19 / 21)$. Moreover, unnecessary RFA was avoided in one case, which was later identified as a pseudolesion based on added CEUS and follow-up imaging findings. Furthermore, therapeutic outcomes including rates 


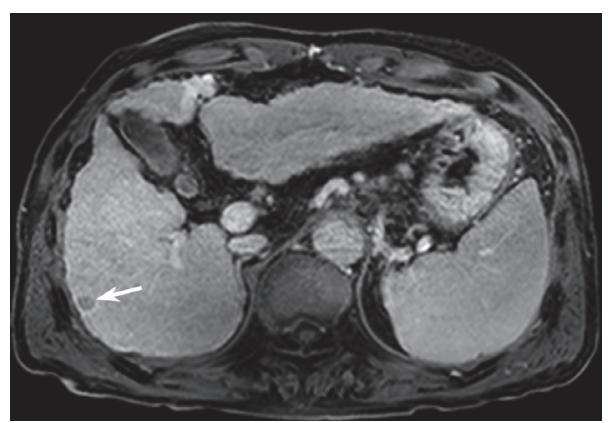

(a) Delayed-phase MRI obtained three minutes after contrast injection showing a 1.1-cm hypointense nodule (arrow) with peripheral rim enhancement in segment 6. Note that the tumor abuts the liver capsule and is located close to the right rib

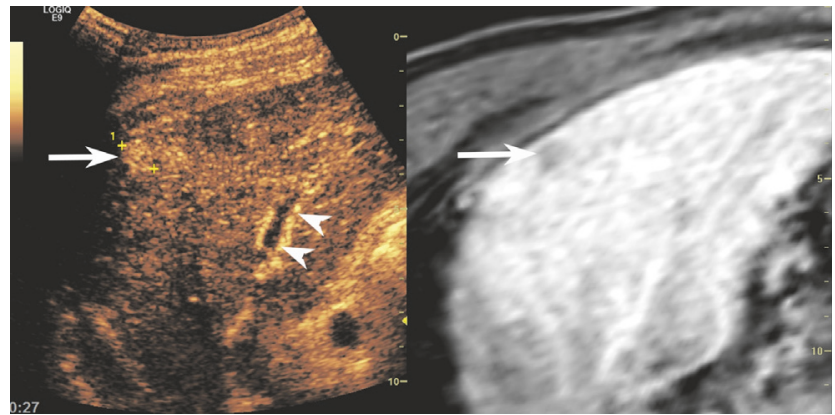

(c) On arterial-phase CEUS, the lesion identified on the previous fusion images did not show arterial enhancement. When the patient breathed in slightly, the liver moved somewhat downwards and the true enhanced lesion (arrow), which was initially located below the right rib, appeared. Note that a portal vein branch (arrowheads) is not seen on US in (b), indicating that the image plane is different from that of (b) even though the US image was obtained at the same intercostal space

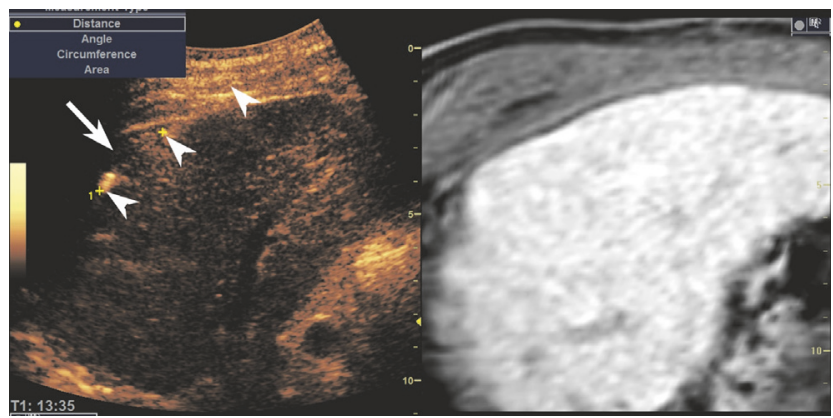

(e) An RF electrode (arrowheads) was positioned in the index tumor (arrow) with confidence under CEUS-added fusion imaging guidance, after which RF energy was applied

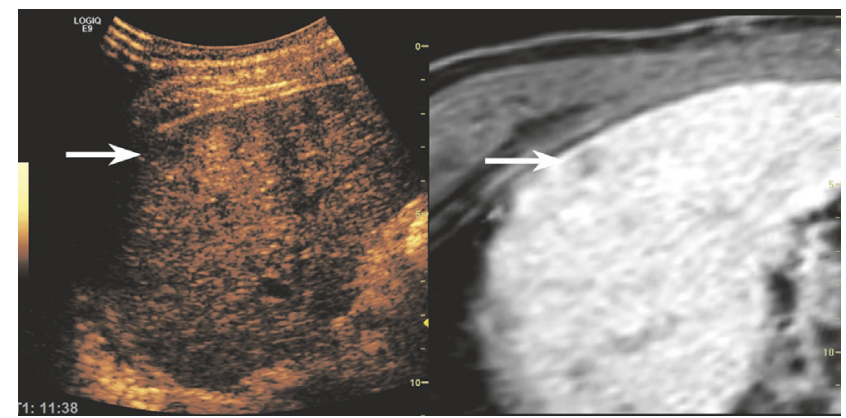

(d) The lesion was seen as a defect (arrow) on the post-vascular phase and the tumor was given a conspicuity score of 1 on CEUS-added fusion imaging

FIGURE 4: Images from a 65-year-old man with a recurrent HCC and hepatitis B virus-related liver cirrhosis who had previously undergone chemoembolization for HCC.

of technical success, primary technique efficacy, major complications, and LTP were excellent. Therefore, we propose that adding CEUS is a valuable strategy for accurate guidance of RFA for small (1-2 cm) HCCs inconspicuous on FI alone.

FI has been used in combination with CEUS for guidance of percutaneous RFA of HCCs in previous studies [10-12, 15, 23]. However, these studies were limited by selection bias

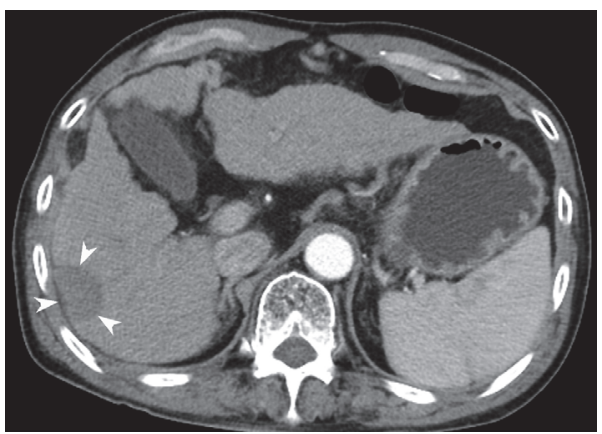

(f) Arterial-phase image of post-RFA CT revealed complete ablation of the tumor with sufficient ablative margins (arrowheads) due to their retrospective nature. Moreover, although they demonstrated the technical feasibility of combined FI and CEUS guidance, few studies have evaluated long-term therapeutic outcomes such as LTP rate [10]. When applying both FI and CEUS, no standardized method has been suggested by any society of interventional radiology or international working group on image-guided tumor ablation. In one 
TABLE 3: Baseline characteristics of 19 patients who underwent percutaneous CEUS-added FI-guided RFA.

\begin{tabular}{|c|c|}
\hline Characteristics & $n=19$ \\
\hline Age (mean $\pm \mathrm{SD}, \mathrm{y})$ (range) & $63.9 \pm 9.3(50-81)$ \\
\hline Sex (female) [number (\%)] & $5(26.3)$ \\
\hline Etiology (HBV/HCV/other) [number (\%)] & $10(52.6) / 2(10.5) / 7(36.8)$ \\
\hline Liver cirrhosis [number (\%)] & $10(52.6)$ \\
\hline \multicolumn{2}{|l|}{ HCC history [number (\%)] } \\
\hline None & $3(15.8)$ \\
\hline Resection & $3(15.8)$ \\
\hline RFA & $2(10.5)$ \\
\hline TACE & $6(31.6)$ \\
\hline Other & $5(26.3)$ \\
\hline Child-Pugh class (A/B) [number (\%)] & $19(100) / 0(0)$ \\
\hline Albumin (mean $\pm \mathrm{SD}, \mathrm{g} / \mathrm{dl})$ & $4.1 \pm 0.4$ \\
\hline Total bilirubin (mean \pm SD, mg/dl) & $1.0 \pm 0.5$ \\
\hline $\mathrm{PT}($ mean $\pm \mathrm{SD}, \mathrm{INR})$ & $1.14 \pm 0.11$ \\
\hline Serum AFP $($ mean \pm SD, ng/ml) & $8.5 \pm 7.3$ \\
\hline Tumor size (mean $\pm \mathrm{SD}, \mathrm{cm}$ ) (range) & $1.2 \pm 0.2(1.0-1.8)$ \\
\hline \multicolumn{2}{|l|}{ Segment [number (\%)] } \\
\hline I & $0(0)$ \\
\hline II & $1(5.3)$ \\
\hline III & $2(10.5)$ \\
\hline IV & $1(5.3)$ \\
\hline $\mathrm{V}$ & $6(31.6)$ \\
\hline VI & $3(15.8)$ \\
\hline VII & $4(21.1)$ \\
\hline VIII & $2(10.5)$ \\
\hline Subcapsular location ${ }^{\mathrm{a}}$ (yes) [numbers (\%)] & $5(26.3)$ \\
\hline Subphrenic location ${ }^{\mathrm{b}}$ (yes) [numbers (\%)] & $5(26.3)$ \\
\hline Time interval between MR imaging and planning US (mean \pm SD, days) (range) & $12.4 \pm 6.8(2-31)$ \\
\hline Time interval between planning US and RFA (mean \pm SD, days) (range) & $12.9 \pm 8.0(0-32)$ \\
\hline \multirow{2}{*}{ Conspicuity score (median, range) } & 3, 3-4 (before CEUS) \\
\hline & $1,1-4$ (after CEUS) \\
\hline Follow-up after RFA (median, range) (months) & $30.2,14.0-36.9$ \\
\hline
\end{tabular}

Data are presented as the number of patients or tumors with percentages in parentheses, unless otherwise specified.

$\mathrm{AFP}=\alpha$-fetoprotein; FI = fusion imaging; HBV = hepatitis $\mathrm{B}$ virus; $\mathrm{HCV}=$ hepatitis $\mathrm{C}$ virus; $\mathrm{INR}=$ international normalized ratio; $\mathrm{PT}=$ prothrombin time; $\mathrm{RFA}=$ radiofrequency ablation; $\mathrm{TACE}=$ transcatheter arterial chemoembolization; $\mathrm{SD}=$ standard deviation.

a Subcapsular location was defined as when the index tumor abutted the liver capsule.

${ }^{\mathrm{b}}$ Subphrenic location was defined as when the index tumor was located within $1 \mathrm{~cm}$ from the diaphragm [6].

TABLE 4: Therapeutic outcomes after CEUS-added FI-guided RFA.

\begin{tabular}{lr}
\hline Outcome & $n=19$ \\
\hline Number of ablation sessions (mean \pm SD) & $1.1 \pm 0.2$ \\
Technical success rate (\%) & $94.7 \%(18 / 19)$ \\
Primary technique efficacy rate (\%) & $100 \%(19 / 19)$ \\
Major complication rate (\%) & $0 \%(0 / 19)$ \\
Local tumor progression rate (\%) & $10.5 \%(2 / 19)$ \\
\hline
\end{tabular}

study, CEUS was applied first, followed by FI [14], whereas in other studies, FI was applied first, followed by CEUS $[11,15,23]$. Both methods have also been used in a single study [10]. Although no data have been obtained to evaluate which method is better, we propose that FI be applied first because it requires no additional cost related to contrast agents in most cases. As demonstrated in our study, many small HCCs can still be identified using only FI. In addition, the overall procedure time of CEUS-guided RFA can be substantially decreased if FI is applied first. 
This is because the location of the target lesion can be estimated based on fused CT/MRI, which obviates the need for a second injection of Sonazoid to obtain the optimal arterial phase of the target lesion [11]. Moreover, when FI is applied first, image fusion can be performed within 3-4 minutes using manual registration methods $[2,5]$. Nowadays, automatic registration methods facilitate easier and faster image fusion $[24,25]$. Therefore, this procedure is much faster than typical CEUS performed using Sonazoid, in which a waiting period of about 10 minutes is needed after the initiation of contrast agent injection to obtain optimal postvascular phase imaging.

Tumor size is one of the most important factors affecting lesion conspicuity on both B-mode US and FI of B-mode US and CT/MRI $[1,16,26]$. As expected, the mean size of tumors inconspicuous on FI was only $1.2 \mathrm{~cm}$ in our cohort. However, the rate of primary technique efficacy after percutaneous CEUS-added FI-guided RFA was 100\% (19/19) and the LTP rate at 3 years was $10.8 \%$. This implies that small HCCs (BCLC stage 0 ) can be ablated effectively once they are accurately localized.

According to a previous study [6], subphrenic or subcapsular tumor location is an important factor affecting mistargeting after FI-guided RFA of HCCs. Peripheral tumor location implies that large anatomic landmarks such as the portal vein branch cannot be used to locate the tumor. In addition, some tumors can be obscured by the rib shadow. Moreover, liver deformation and movement due to a patient's breathing or heart beat may be more apparent in peripheral liver than in central liver $[6,27]$. This technical difficulty of percutaneous RFA of tumors in peripheral locations was also observed in our study. Residual tumor was noted in one patient with a tumor located in the subcapsular area, just below the right rib. Nevertheless, mistargeting or incomplete ablation can be avoided after CEUS, as seen in one of our cases (Figure 4).

Our study did have several limitations. First, this was a single-center study conducted at a tertiary academic hospital with a large volume of RFAs. In general, outcomes after RFA depend on operator experience. Also, 75\% (66/88) of the study population had hepatitis B-related liver disease; thus, the results of this study may not be generalizable to other institutions or countries where hepatitis B virus is not the primary cause of liver cirrhosis or HCC. Second, lesion conspicuity was graded by one of three experienced radiologists and interobserver agreement was not assessed because the conspicuity score was graded in a prospective manner at the time of US examination. Third, more patients were excluded than expected before initiating the study. For example, four patients in whom artificial ascites was used during RFA were excluded from our final patient population because artificial ascites can enhance lesion conspicuity by improving the sonographic window [28]. Since we sought to determine the direct effect of CEUS on lesion conspicuity during percutaneous FI-guided RFA, this exclusion criterion may not have significantly affected our study outcome. Fourth, CEUS was selectively applied to a small number of patients with HCCs inconspicuous on FI alone because FI was sufficient for guidance of RFA of HCCs in many cases $[5,16]$. Therefore, comparing the rate of conspicuous HCCs between FI alone and CEUS-added FI may yield limited insight. Applying CEUS to the entire study sample could have provided a more comprehensive understanding of the advantages and disadvantages of combined FI and CEUS.

In conclusion, adding CEUS to FI significantly improved the conspicuity of HCCs inconspicuous on FI alone, which enabled direct targeting for percutaneous RFA. This led to successful percutaneous RFA with excellent therapeutic outcomes.

\section{Data Availability}

The data used to support the findings of this study are available from the corresponding author upon request.

\section{Conflicts of Interest}

The authors declare that they have no conflicts of interest.

\section{Acknowledgments}

This study was supported in part by a grant from GE Healthcare.

\section{References}

[1] M. W. Lee, Y. J. Kim, H. S. Park et al., "Targeted sonography for small hepatocellular carcinoma discovered by CT or MRI: Factors affecting sonographic detection," American Journal of Roentgenology, vol. 194, no. 5, pp. W396-W400, 2010.

[2] J. H. Min, M. W. Lee, H. Rhim et al., "Radiofrequency ablation for viable hepatocellular carcinoma around retained iodized oil after transcatheter arterial chemoembolization: Usefulness of biplane fluoroscopy plus ultrasound guidance," Korean Journal of Radiology, vol. 13, no. 6, pp. 784-794, 2012.

[3] G. Mauri, L. Cova, S. De Beni et al., "Real-Time US-CT/MRI Image Fusion for Guidance of Thermal Ablation of Liver Tumors Undetectable with US: Results in 295 Cases," CardioVascular and Interventional Radiology, vol. 38, no. 1, pp. 143-151, 2015.

[4] Y. Minami, H. Chung, M. Kudo et al., "Radiofrequency ablation of hepatocellular carcinoma: Value of virtual CT sonography with magnetic navigation," American Journal of Roentgenology, vol. 190, no. 6, pp. W335-W341, 2008.

[5] S. J. Ahn, J. M. Lee, D. H. Lee et al., "Real-time US-CT/MR fusion imaging for percutaneous radiofrequency ablation of hepatocellular carcinoma," Journal of Hepatology, vol. 66, no. 2, pp. 347-354, 2017.

[6] S. Lim, M. W. Lee, H. Rhim et al., "Mistargeting after fusion imaging-guided percutaneous radiofrequency ablation of hepatocellular carcinomas," Journal of Vascular and Interventional Radiology, vol. 25, no. 2, pp. 307-314, 2014.

[7] R. Masuzaki, S. Shiina, R. Tateishi et al., "Utility of contrastenhanced ultrasonography with Sonazoid in radiofrequency ablation for hepatocellular carcinoma," Journal of Gastroenterology and Hepatology, vol. 26, no. 4, pp. 759-764, 2011.

[8] Y. Minami, M. Kudo, K. Hatanaka et al., "Radiofrequency ablation guided by contrast harmonic sonography using perfluorocarbon microbubbles (Sonazoid) for hepatic malignancies: An 
initial experience," Liver International, vol. 30, no. 5, pp. 759764, 2010.

[9] M. Kudo, K. Hatanaka, and K. Maekawa, "Newly developed novel ultrasound technique, defect reperfusion ultrasound imaging, using Sonazoid in the management of hepatocellular carcinoma," Oncology, vol. 78, no. 1, pp. 40-45, 2010.

[10] T. Minami, Y. Minami, H. Chishina et al., "Combination guidance of contrast-enhanced us and fusion imaging in radiofrequency ablation for hepatocellular carcinoma with poor conspicuity on contrast-enhanced US/fusion imaging," Oncology (Switzerland), vol. 87, pp. 55-62, 2014.

[11] J. H. Y. Min, H. K. E. Lim, S. Lim et al., "Radiofrequency ablation of very-early-stage hepatocellular carcinoma inconspicuous on fusion imaging with B-mode US: value of fusion imaging with contrast-enhanced US," Clinical and Molecular Hepatology, vol. 20, no. 1, pp. 61-70, 2014.

[12] X.-W. Bo, H.-X. Xu, D. Wang et al., "Fusion imaging of contrastenhanced ultrasound and contrast-enhanced CT or MRI before radiofrequency ablation for liver cancers," British Journal of Radiology, vol. 89, no. 1067, Article ID 20160379, 2016.

[13] G. Mauri, E. Porazzi, L. Cova et al., "Intraprocedural contrastenhanced ultrasound (CEUS) in liver percutaneous radiofrequency ablation: Clinical impact and health technology assessment," Insights into Imaging, vol. 5, no. 2, pp. 209-216, 2014.

[14] Y. Dong, W.-P. Wang, F. Mao, Z.-B. Ji, and B.-J. Huang, "Application of imaging fusion combining contrast-enhanced ultrasound and magnetic resonance imaging in detection of hepatic cellular carcinomas undetectable by conventional ultrasound," Journal of Gastroenterology and Hepatology, vol. 31, no. 4, pp. 822-828, 2016.

[15] E. M. Jung, C. Friedrich, P. Hoffstetter et al., "Volume navigation with contrast enhanced ultrasound and image fusion for percutaneous interventions: First results," PLoS ONE, vol. 7, no. 3, Article ID e33956, 2012.

[16] M. W. Lee, H. Rhim, D. Ik Cha, Y. Jun Kim, and H. K. Lim, "Planning US for percutaneous radiofrequency ablation of small hepatocellular carcinomas $(1-3 \mathrm{~cm})$ : Value of fusion imaging with conventional US and CT/MR Images," Journal of Vascular and Interventional Radiology, vol. 24, no. 7, pp. 958-965, 2013.

[17] H. Rhim, D. Choi, Y.-S. Kim, H. K. Lim, and B.-K. Choe, "Ultrasonography-guided percutaneous radiofrequency ablation of hepatocellular carcinomas: A feasibility scoring system for planning sonography," European Journal of Radiology, vol. 75, no. 2, pp. 253-258, 2010.

[18] H. Rhim, H. L. Mi, Y.-S. Kim, D. Choi, J. L. Won, and H. K. Lim, "Planning sonography to assess the feasibility of percutaneous radiofrequency ablation of hepatocellular carcinomas," American Journal of Roentgenology, vol. 190, no. 5, pp. 1324-1330, 2008.

[19] J.-E. Kim, Y.-S. Kim, H. Rhim et al., "Outcomes of patients with hepatocellular carcinoma referred for percutaneous radiofrequency ablation at a tertiary center: Analysis focused on the feasibility with the use of ultrasonography guidance," European Journal of Radiology, vol. 79, no. 2, pp. e80-e84, 2011.

[20] J. Bruix and M. Sherman, "Management of hepatocellular carcinoma: an update," Hepatology, vol. 53, no. 3, pp. 1020-1022, 2011.

[21] M. Ahmed, L. Solbiati, C. L. Brace et al., "Image-guided tumor ablation: standardization of terminology and reporting criteria-a 10-year update," J Vasc Interv Radiol, vol. 25, no. 11, pp. 1691-1705, 2014.

[22] M. W. Lee, H. Rhim, D. I. Cha et al., "Percutaneous radiofrequency ablation of hepatocellular carcinoma: Fusion imaging guidance for management of lesions with poor conspicuity at conventional sonography," American Journal of Roentgenology, vol. 198, no. 6, pp. 1438-1444, 2012.

[23] M. W. Lee, "Fusion imaging of real-time ultrasonography with CT or MRI for hepatic intervention," Ultrasonography, vol. 33, no. 4, pp. 227-239, 2014.

[24] G. Mauri, S. De Beni, L. Forzoni et al., "Virtual navigator automatic registration technology in abdominal application," Conference proceedings : ... Annual International Conference of the IEEE Engineering in Medicine and Biology Society. IEEE Engineering in Medicine and Biology Society. Annual Conference, vol. 2014, pp. 5570-5574, 2014.

[25] D. I. Cha, M. W. Lee, A. Y. Kim et al., "Automatic image fusion of real-time ultrasound with computed tomography images: A prospective comparison between two auto-registration methods," Acta Radiologica, vol. 58, no. 11, pp. 1349-1357, 2017.

[26] Y. Kunishi, K. Numata, M. Morimoto et al., "Efficacy of fusion imaging combining sonography and hepatobiliary phase MRI with Gd-EOB-DTPA to detect small hepatocellular carcinoma," American Journal of Roentgenology, vol. 198, no. 1, pp. 106-114, 2012.

[27] M. W. Lee, H. J. Park, T. W. Kang et al., "Image Fusion of RealTime Ultrasonography with Computed Tomography: Factors Affecting the Registration Error and Motion of Focal Hepatic Lesions," Ultrasound in Medicine \& Biology, vol. 43, no. 9, pp. 2024-2032, 2017.

[28] H. Rhim, H. K. Lim, Y.-S. Kim, and D. Choi, "Percutaneous radiofrequency ablation with artificial ascites for hepatocellular carcinoma in the hepatic dome: Initial experience," American Journal of Roentgenology, vol. 190, no. 1, pp. 91-98, 2008. 


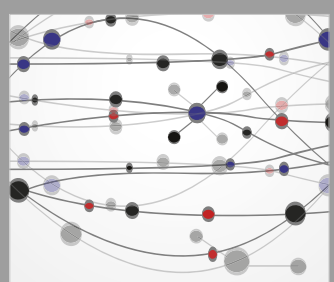

The Scientific World Journal
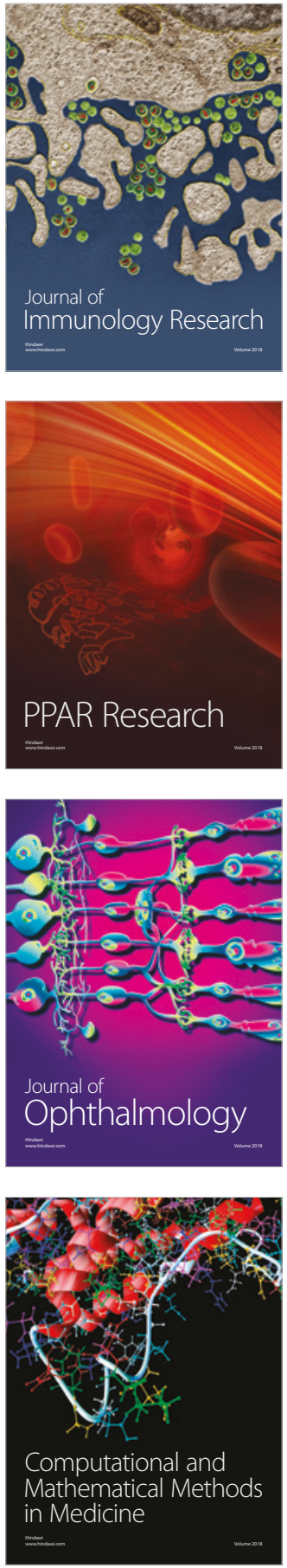

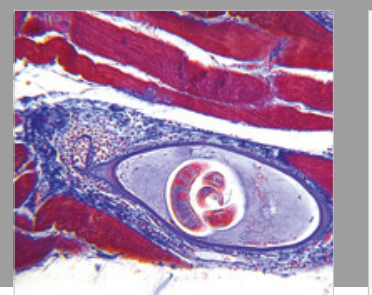

Gastroenterology Research and Practice

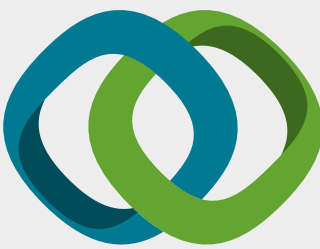

\section{Hindawi}

Submit your manuscripts at

www.hindawi.com
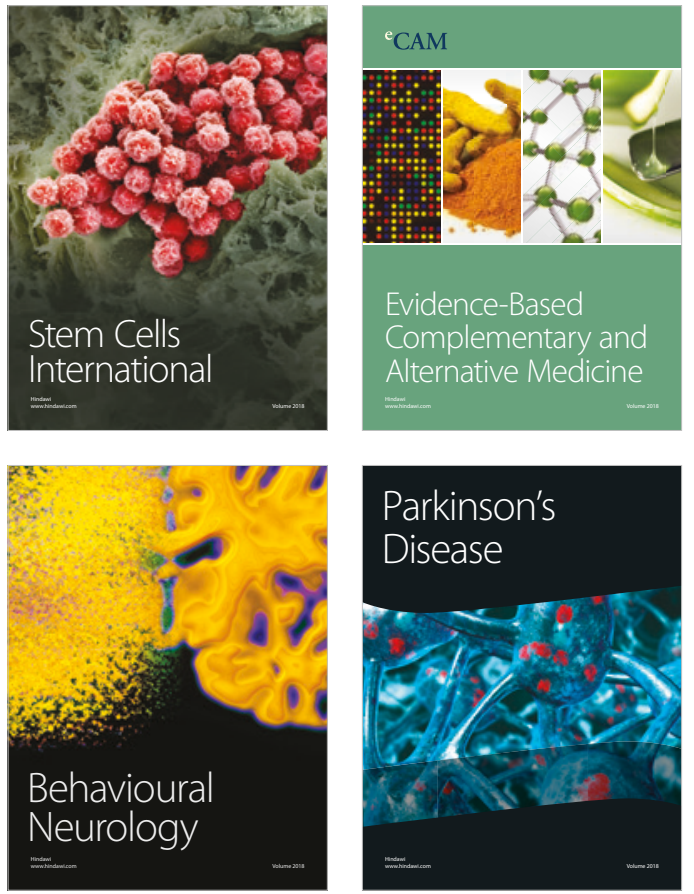

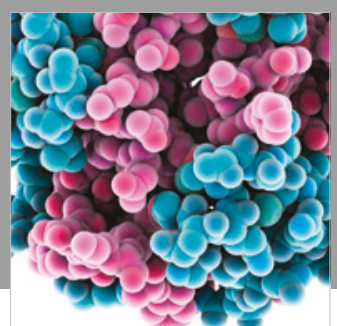

ournal of

Diabetes Research

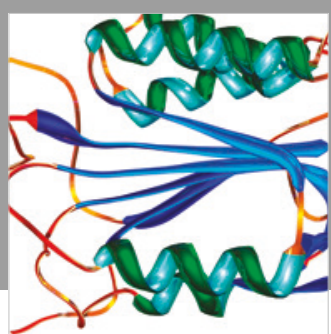

Disease Markers
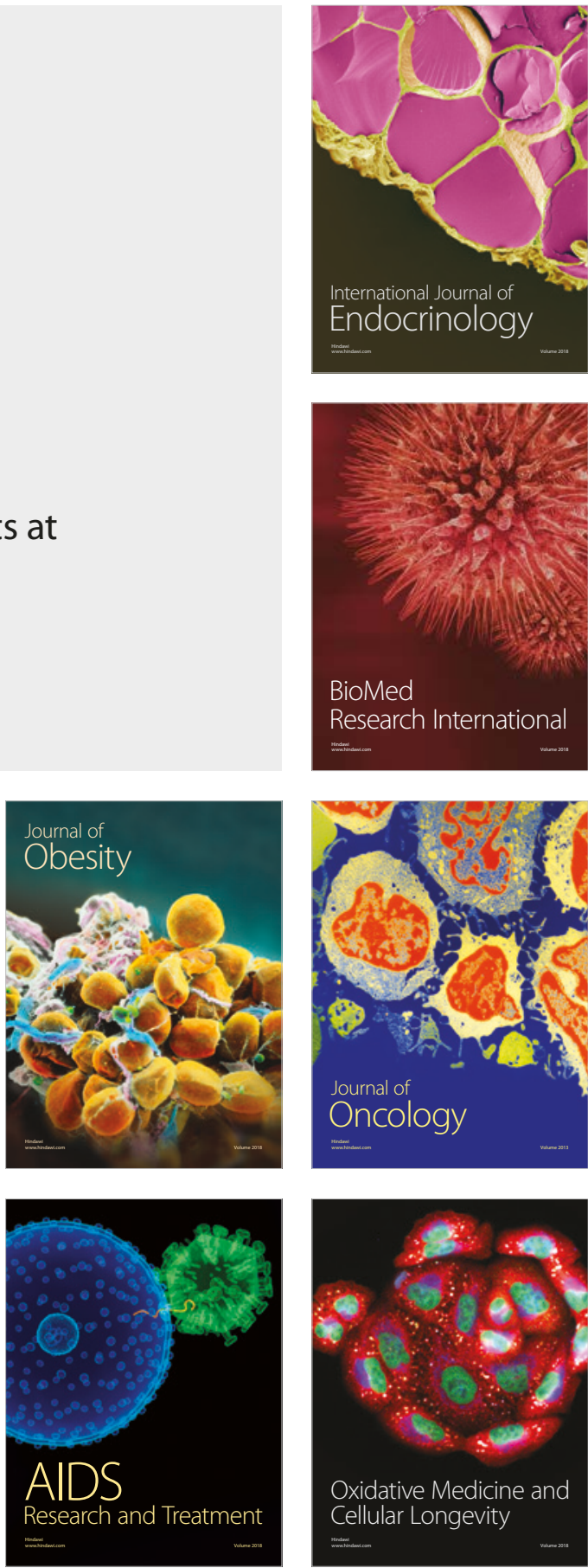